\title{
Software Development Outsourcing Relationships Trust: A Systematic Literature Review Protocol
}

\author{
Siffat Ullah Khan \\ School of Computing and Mathematics, \\ Keele University, UK \\ s.khan@epsam.keele.ac.uk
}

\author{
Mahmood Niazi \\ School of Computing and Mathematics, \\ Keele University, UK \\ mkniazi@cs.keele.ac.uk
}

\author{
Naveed Ikram \\ International Islamic University, \\ Islamabad, Pakistan \\ Naveed.ikram@iiu.edu.pk
}

\begin{abstract}
CONTEXT - Trust plays a vital role in establishing and maintenance of the outsourcing relationships between client and vendor organisations.

OBJECTIVE - This paper aims to review the literature in a systematic way to identify the factors that can have a positive impact on trust building and its maintenance between the clients and vendors organisations in the context of offshore software outsourcing relationships.

METHOD - We have developed a systematic literature review (SLR) protocol for software outsourcing relationships trust (SORT). We are in the process of implementing the protocol for SORT.

EXPECTED OUTCOME - The anticipated outcome of this review will be a list of factors which can play an important role in the establishment and maintenance of trust in software outsourcing relationships.
\end{abstract}

Systematic Literature Review, Software Development Outsourcing, Trust, Client-Vendor Relationship

\section{INTRODUCTION}

Trust is one of the important factors for effectively managing software development outsourcing relationships (Ali-Babar et al, 2007). This paper describes the systematic literature review (SLR) (Kitchenham, 2004) protocol which will be used to investigate the factors that can have a positive or negative impact on trust building and its maintenance between the clients and vendors organisations. The goal of our research is to gain an in depth understanding of factors that facilitate the formation and maintenance of trust between client and vendor organisations in the context of offshore software outsourcing relationships.

Different studies show that trust is critical for all business relationships (Kanawattanachai and Yoo, 2002), however the focus of our study is on software development outsourcing relationships (Oza et al, 2006). Over the last decade, many firms in the USA and UK have outsourced software development projects to offshore countries. The developed countries are outsourcing projects to developing countries to take advantage from the reduced labour costs and economies of scale. More than $50 \%$ of the American Fortune 500 firms and an increasing proportion of European and Japanese firms are users of offshore software outsourcing (Sahay et al, 2003). Understanding different factors in software development outsourcing relationships can help to ensure the successful outcome of outsourcing projects and long lasting relationships between clients and vendors (Ali-Babar et al, 2007; Goo et al, 2007).

The paper reports our study plan in the form of SLR protocol. This paper presents the design of a first of its kind study in software outsourcing relationships trust. There are two research questions that have motivated the work reported in this paper (Ali-Babar et al, 2007):

RQ1: What factors are important for establishing trust in offshore software outsourcing relationships?

RQ2: What factors are important for maintaining and strengthening trust in offshore software outsourcing relationships?

\section{BACKGROUND}

Software development outsourcing is a contract-based relationship where a company (client) contracts out its software development activities to another company (vendor), who provides services for remuneration (AliBabar et al, 2007). Previous work suggests that half of the companies that have tried outsourcing have failed to realise the anticipated results (Bradstreet, 2000). There are many reasons for outsourcing failures (Cataldo et al, 2007; Mary and Joseph, 2009). One of the major issues in outsourcing is that many clients endorse outsourcing contracts with their vendors prior to test their readiness for the outsourcing activity (Bradstreet, 2000; Khan et al, 2009). Other reasons include a lack of software development outsourcing best practices and difficulties in creating confidence and trust among the outsourcing companies (Cataldo et al, 2007; Oza et al, 2006; Stark et al, 2006). 
However, despite the importance of the global software development, little empirical research has been carried out on software development outsourcing practices in general and building confidence and trust among the outsourcing companies in particular.

A number of researchers have tried to address some of the issues of software development outsourcing, e.g. (Nguyen et al.(Nguyen et al, 2006); Oza et al. (Oza et al, 2006), Sabherwal (Sabherwal, 1999),).

The work in this paper complements work previously done in these studies. However, nobody has used the Systematic Literature Review (SLR) (EBSE-TechnicalReport, 2007) approach in order to identify important factors for establishing and maintaining trust in offshore software outsourcing relationships. A SLR is a defined and methodical way of identifying, assessing, and analysing published primary studies in order to investigate a specific research question (EBSETechnical-Report, 2007; Khan et al, 2009; Staples and Niazi, 2007). In finding, evaluating, and summarising all available evidence on software development outsourcing trust, a SLR will provide a greater level of validity in its findings than might be possible in any one of the existing studies discussed above. A SLR protocol designed has been discussed in this paper.

\section{SYSTEMATIC LITERATURE REVIEW PROTOCOL FOR SOFTWARE DEVELOPMENT OUTSOURCING TRUST}

Kitchenham describes the three main phases of a systematic review process: planning the review, conducting the review, and reporting the review (EBSETechnical-Report, 2007). In this section we describe the first phase of a systematic review (i.e. planning the review). The output from this phase is a systematic review protocol that defines the purpose and procedures for the review. Kitchenham notes that a pre-defined protocol is necessary to reduce the possibility of researcher bias (EBSE-Technical-Report, 2007).

\subsection{Search Strategy}

- Identifying Search Terms

The following search strategy is used for the construction of search terms.

a) Use the Research Questions for the derivation of major terms, by identifying population, intervention and outcome;

b) For these major terms, find the alternative spellings and synonyms;

c) Verify the key words in any relevant paper;

d) Use of Boolean Operators for conjunction if the database allows, in such a way, to use 'OR' operator for the concatenation of alternative spellings and synonyms whereas 'AND' for the concatenation of major terms.

\section{Results for a)}

RQ1: offshore software outsourcing relationships, factors, establishing trust

RQ2: offshore software outsourcing relationships, factors, trust maintenance, strengthening trust

\section{Results for $b$ )}

(i) RQ1:

Offshore software outsourcing: ("offshore software outsourcing" OR "information systems outsourcing" OR "information technology outsourcing" OR "IS outsourcing" OR "IT outsourcing" OR "CBIS outsourcing" OR "computer-based information systems outsourcing" OR "software facility management" OR "software contracting-out" OR "multi-site software development" OR "distributed software development")

Trust: (trust OR trustworthy OR trustworthiness OR trusted OR reliance OR "relationship building" OR "relationship maintenance" OR reputation OR satisfaction OR reliable OR reliability OR "best performance" OR "expectations match" OR responsiveness OR "good track record" OR "project success" OR co-ordination OR association)

Factors: (factors OR drivers OR motivators OR elements OR characteristics OR parameters)

Establishing: (Establishing OR establishment OR setting-up, building, launching, creating, agreement OR contracting OR alliance OR approval OR pleasure OR sure OR confidence OR "successful relationship" OR "relationship management" OR satisfaction)

(ii) RQ2:

Offshore software outsourcing: ("offshore software outsourcing" OR "information systems outsourcing" OR "information technology outsourcing" OR "IS outsourcing" OR "IT outsourcing" OR "CBIS outsourcing" OR "computer-based information systems outsourcing" OR "software facility management" OR "software contracting-out" OR "multi-site software development" OR "distributed software development") Trust: (Trust OR trustworthy $\mathrm{R}$ trustworthiness OR trusted OR reliance OR "relationship building" OR "relationship maintenance" OR reputation OR satisfaction OR reliable OR reliability OR best performance OR "expectations match" OR responsiveness OR "good track record" OR "project success" OR co-ordination OR association)

Factors: (factors OR drivers OR motivators OR elements OR characteristics OR parameters)

Maintenance: (maintenance OR maintaining OR preserve OR sustain OR retain OR uphold OR continue OR keeping- up)

Strengthening: (strengthening OR strengthen OR fortification OR supporting OR reinforcing OR reinforcement) 


\section{Results for c)}

Offshore software outsourcing relationships, Trust in software outsourcing relationship, trust building, maintaining trust, outsourcing alliance, software outsourcing vendor

\section{Results for d)}

RQ1.

("Offshore software outsourcing" OR "information systems outsourcing" OR "information technology outsourcing" OR "IS outsourcing" OR "IT outsourcing" OR "CBIS outsourcing" OR "computer-based information systems outsourcing" OR "software facility management" OR "software contracting-out" OR "multisite software development" OR "distributed software development") AND (Trust OR trustworthy OR trustworthiness OR trusted OR reliance OR "relationship building" OR "relationship maintenance" OR reputation OR satisfaction OR reliable OR reliability OR best performance OR "expectations match" OR responsiveness OR "good track record" OR "project success" OR co-ordination OR association) OR (Factors OR drivers OR motivators OR elements OR characteristics OR parameters) OR (Establishing OR establishment OR setting-up, building, launching, creating, agreement OR contracting OR alliance OR approval OR pleasure OR sure OR confidence OR "successful relationship" OR "relationship management" OR satisfaction))

RQ2.

("Offshore software outsourcing" OR "information systems outsourcing" OR "information technology outsourcing" OR "IS outsourcing" OR "IT outsourcing" OR "CBIS outsourcing" OR "computer-based information systems outsourcing" OR "software facility management" OR "software contracting-out" OR "multisite software development" OR "distributed software development") AND (Trust OR trustworthy R trustworthiness OR trusted OR reliance OR "relationship building" OR "relationship maintenance" OR reputation OR satisfaction OR reliable OR reliability OR best performance OR "expectations match" OR responsiveness OR "good track record" OR "project success" OR co-ordination OR association) OR (Factors OR drivers OR motivators OR elements OR characteristics OR parameters) OR (Maintenance OR maintaining OR preserve OR sustain OR retain OR uphold OR continue OR keeping- up) OR (Strengthening OR strengthen OR fortification OR supporting OR reinforcing OR reinforcement))

\section{- Resources to be searched}

IEEE Xplore, ACM Portal, ScienceDirect, CiteSeer Digital Library and Google Scholar.

\section{- Search Constraints and Validation}

We will search all published papers related to our research questions and will not put any date boundaries. A prior search was conducted using the following set of major terms and we found certain relevant papers in CiteSeer digital libraries. ("Offshore software outsourcing" OR "IT outsourcing" OR "IS/IT outsourcing") AND (trust OR relationship OR contract OR "vendor's reputation" OR factors OR "trust building" OR "maintaining trust").

\subsection{Publication Selection \\ - Inclusion Criteria}

The following criteria were used to determine which piece of literature found by the search term will be used for the data extraction.

- Studies that describe factors/motivators for offshore software outsourcing trust building/ trust development

- Studies that describe factors/motivators for offshore software outsourcing trust maintenance/ strengthening

- Studies that describe criteria for a successful software outsourcing relationships

\section{- Exclusion Criteria}

The following criteria were used to determine which piece of literature found by the search term will be excluded.

- Studies that are not relevant to the research questions and Studies that do not describe software outsourcing relationships

\section{- Selecting Primary Sources}

Initial selection of the primary sources will be performed by reviewing the title, keywords and abstract. The purpose is to exclude/ignore only those results which have no relevance to the problem/research questions. The primary sources chosen in the initial selection process will be checked against the aforesaid inclusion/exclusion criteria by reviewing carefully through full text of the studies. The source will be sent to the secondary reviewer, for review in case of any uncertainty regarding the inclusion or exclusion decision.

\subsection{Publication Quality Assessment}

The measurement of quality will be performed after final selection of publications. The quality checklist contains the following questions:

- Is it clear how the factors for establishing/building trust between clients and vendors were identified in offshore software outsourcing relationship?

- Is it clear how the factors for maintaining and strengthening trust between clients and vendors were identified in offshore software outsourcing relationship?

Each of the above factors will be marked as 'YES', 'NO', 'Partially' or N.A'. A secondary reviewer will score a small subset for validation. 


\subsection{Data Extraction Strategy}

The review will be undertaken by a single researcher, who will be responsible for the data extraction. A secondary reviewer will be approached for guidance in case of an issue regarding the data extraction.

The secondary reviewer will select few publications randomly from the list of publication already chosen by the primary reviewer. The secondary reviewer will independently extract data from the randomly selected publications. The results will then be compared with the results produced by the primary reviewer.

\subsection{Data Synthesis}

Due to two research questions, the synthesis will also be categorized into two parts. For the Research Question1, the data will be synthesized by creating one summary table having the columns (S.No, Factors, Frequency, Percentages) showing the list of all the factors along with their frequencies and percentages. The complete detail of every factor mentioned in the summary table will be recorded in a separate table which will hold the following columns (Factor group name, S.No of reference, factor's subgroups, paper reference/paper title). For the Research Question2, the same process will be performed as for the RQ1 mentioned above.

\section{CONCLUSIONS AND FUTURE WORK}

Gallivan and Oh (1999) have grouped the offshore outsourcing relationships into four different types, based on the number of clients and vendors involved in the outsourcing contract. These are Simple Dyadic Relationships, Multi-Vendors Relationships, CoSourcing Relationships and Complex Relationships. Trust plays a vital role in establishing and maintenance of all these four types of relationships. Previously, no systematic literature process has been performed in order to identify factors which are important for establishing and maintaining trust in offshore software outsourcing relationships. In this paper we discussed our study plan in the form of the SLR protocol. In our future work we will implement this protocol and the findings of this research will shed some light on the factors that should be considered critical during establishing and maintaining trust in offshore software outsourcing relationships.

\section{REFERENCES}

Ali-Babar, M., Verner, J. and Nguyen, P. 2007. Establishing and maintaining trust in software outsourcing relationships: An empirical investigation, The Journal of Systems and Software 80 (2007). 14381449.

Bradstreet, D. 2000. Dun \& Bradstreet's Barometer of Global Outsourcing, Dun \& Bradstreet, http://findarticles.com/p/articles/mi m0EIN/is $2000 \mathrm{Fe}$ b 24/ai 59591405, September, 2007.

Cataldo, M., Bass, M., Herbsleb, D., J. and Bass, L. 2007. On Coordination Mechanisms in Global Software Development. International Conference on Global Software Engineering. 71-80.

EBSE-Technical-Report. 2007. Guidelines for performing Systematic Literature Reviews in Software Engineering, EBSE Technical Report: EBSE-2007-01. http://www.dur.ac.uk/ebse/resources/guidelines/Syste matic-reviews-5-8.pdf.

Gallivan, M. J. and Oh, W. 1999. Analyzing IT Outsourcing Relationships as Alliances among Multiple Clients and Vendors. Proceedings of the 32nd Hawaii international Conference on System Sciences. Hawaii, USA.

Goo, J., Kishore, R., Nam, K., Rao, H., R. and Song, Y. 2007. An investigation of factors that influence the duration of IT outsourcing relationships, Decision Support Systems 42 (4). 2107-2125.

Kanawattanachai, P. and Yoo, Y. 2002. Dynamic nature of trust in virtual teams, Journal of Strategic Information Systems 11 (3-4). 187-213.

Khan, S., Niazi, M. and Ahmad, R. 2009. Critical Success Factors for Offshore Software Development Outsourcing Vendors: A Systematic Literature Review. The 4th IEEE International Conference on Global Software Engineering. Ireland.

Kitchenham, B. 2004. Procedures for Performing Systematic Reviews. Keele University, Technical ReportTR/SE0401,

Mary, L. and Joseph, R. 2009. Effects of offshore outsourcing of information technology work on client project management, Strategic Outsourcing: An International Journal 2 (1). 4-26.

Nguyen, P., Ali-Babar, M. and Verner, J. 2006. Trust in software outsourcing relationships: an analysis of Vietnamese practitioners' views. EASE. 10-19.

Oza, N. V., Hall, T., Rainer, A. and Grey, S. G. 2006. Trust in software outsourcing relationships: An empirical investigation of Indian software companies, Information \& Software Technology 48 (5). 345-354.

Sabherwal, R. 1999. The role of trust in outsources IS development projects,, Communication of ACM 4280 86.

Sahay, S., Nicholson, B. and Krishna, S. 2003. Global IT outsourcing. Cambridge University Press.,

Staples, M. and Niazi, M. 2007. Experiences Using Systematic Review Guidelines, Journal of Systems and Software. 80 (9). 1425-1437.

Stark, J., Arlt, M. and Walker, D., H. T. 2006. Outsourcing Decisions and Models - Some Practical Considerations for Large Organizations. International Conference on Global Software Engineering. 12-17. 